Phyl ogenet i c exami nat i on of crude drugs der i ved from Yunnanese Swerti a pl ant s

\begin{tabular}{|l|l|}
\hline 著者 & $\begin{array}{l}\text { Kaki uchi Nobuko, I waki Naoko, M kage Nasayuki , } \\
\text { Xi ao Huai, hang Zhi gang, Hat tor i Nasao }\end{array}$ \\
\hline $\begin{array}{l}\text { j our nal or } \\
\text { publ i cat i on t i t l e }\end{array}$ & Jour nal of Nat ur al Medi ci nes \\
\hline vol une & 68 \\
\hline number & 1 \\
\hline page range & $206-210$ \\
\hline year & $2014-01-01$ \\
\hline URL & ht t p: //hdl . handl e. net /2297/37011 \\
\hline
\end{tabular}




\title{
Phylogenetic examination of crude drugs derived from Yunnanese Swertia plants
}

\author{
Nobuko Kakiuchi $\cdot$ Naoko Iwaki • \\ Masayuki Mikage $\cdot$ Huai Xiao $\cdot$ \\ Zhigang Wang $\cdot$ Masao Hattori
}

Received: 12 January 2013/Accepted: 10 April 2013/Published online: 8 May 2013

(C) The Japanese Society of Pharmacognosy and Springer Japan 2013

\begin{abstract}
Aiming to examine whether the genetic background of the crude drugs derived from four Yunnanese Swertia plants and their chemical constituent profiles correlate, we analyzed the nucleotide sequences of their nuclear ribosomal DNA regions including ITS1, 5.8S ribosomal RNA gene, and ITS2, together with those of Japanese S. japonica and S. pseudochinensis from Hebei Province. The result that two of the Yunnanese Swertia plants, S. binchuanensis and S. punicea, were genetically similar may explain their similarity in chemical constituent profiles. On the other hand, in spite of differences in chemical profile, S. decora and S. pseudochinensis were genetically close. The other Yunnanese Swertia plants, $S$. delavayi, and S. japonica, stood at intermediate positions between these two genetically similar pairs. The result suggests that although genetic background would have an influence, environmental factors, e.g., soil and weather
\end{abstract}

\section{Present Address:}

N. Kakiuchi $(\square)$

School of Pharmaceutical Sciences, Kyusyu University of Health and Welfare, 1714-1 Yoshino-cho, Nobeoka,

Miyazaki 882-8508, Japan

e-mail: kakiuchi@phoenix.ac.jp

N. Kakiuchi - N. Iwaki · M. Mikage

School of Pharmacy, College of Medical, Pharmaceutical and Health Sciences, Kanazawa University, Kakumacho, Kanazawa 920-1192, Japan

H. Xiao

Department of Pharmacy, Dali University, Dali 671000,

Yunnan Province, China

Z. Wang - M. Hattori

Division of Metabolic Engineering, Institute of Natural

Medicine, University of Toyama, 2630 Sugitani,

Toyama 930-0194, Japan conditions, might be critical for their production of secondary metabolites.

Keywords Swertia · Yunnan - Ribosomal DNA . Phylogeny

\section{Introduction}

Swertia herb derived from various Swertia plants is widely used as a folk medicine in Asian countries including China, India, Korea, and Japan. The crude drug derived from $S$. japonica Makino, for example, is a notable Japanese folk medicine traditionally applied for various purposes such as a cure for stomach-ache and digestive disorders. Recent research has shown that $S$. japonica has a significant bloodsugar-lowering effect and a protective effect on liver cells in animal models $[1,2,3]$. These biological effects are attributable to the major and characteristic groups of the chemical constituents of Swertia plants (xanthones, iridoids and flavonoids). Recently, chemical constituent profiling of the crude drugs derived from four Yunnanese Swertia plants and Japanese S. japonica as well as S. pseudochinensis Hara from Hebei Province was conducted using LC/MS/MS [4]. The results showed the following similarities and differences in chemical profile of the major constituents of the 6 species: (1) S. japonica and S. pseudochinensis showed high swertiamarin content and universal occurrence of irridoids and flavonoids, (2) S. decora Franch and S. delevayi Franch showed high swertiamarin but very low xanthones and flavonoids content, (3) S. binchuanensis T. N. Ho \& S. W. Liu and S. punicea Hensl had relatively high flavonoids content. Aiming to find out whether their genetic background and chemical constituent profile correlate, we analyzed the nucleotide sequences of nuclear ribosomal DNA 
regions including ITS1, 5.8S ribosomal RNA gene, and ITS2 of these 6 Swertia plants.

\section{Materials and methods}

Plant and crude drug materials

Materials used in this study are summarized in Table 1; they were the same materials used in a previous study [4]. Cultivated Swertia japonica specimens were collected in Nagano Prefecture, Japan. Crude drug materials 2, 3, 4, and 5 were obtained from Yunnan Province, China, and were identified as Swertia decora Franch, Swertia binchuanensis T. N. Ho \& S. W. Liu, Swertia punicea Hensl, and Swertia delevayi Franch, respectively, by Prof. Tingnong $\mathrm{He}$ (Northwest Institute of Plateau Biology, Chinese Academy of Science). A crude drug material 6, Swertia pseudochinensis Hara (collected in Hebei Province, China), was supplied by Alps Pharmaceutical Ind. Co. Ltd., Japan. The vouchers were deposited in the herbarium of the School of Pharmacy, Kanazawa University.

\section{Isolation of total DNA}

A single stem of each material $(20-50 \mathrm{mg})$ was frozen in liquid nitrogen and ground into fine powder. Total DNA isolation from the powder was performed using a DNeasy Plant Mini Kit (Qiagen) according to the manufacturer's protocol with minor modifications.

\section{PCR amplification}

The ITS region was amplified by polymerase chain reaction (PCR) using 30-100 ng of total DNA as a template in $25 \mu \mathrm{l}$ of reaction mixture containing $2.5 \mu \mathrm{l}$ of $10 \times \mathrm{PCR}$ buffer for KOD-Plus, $0.2 \mathrm{mM}$ of each dNTP, $1.0 \mathrm{mM}$ of $\mathrm{MgSO}_{4}, 0.5$ units KOD-Plus-polymerase (Toyobo), and $0.4 \mathrm{mM}$ of primers Swer ITS1F (GAG GTC GCG AGA AGT CCA CT) and Swer ITS 1R (CTC AGC GGG TAA TCC CGC CT). Amplification was carried out under the following conditions: pre-heating at $94{ }^{\circ} \mathrm{C}$ for $2 \mathrm{~min}$; 30 cycles of denaturation at $94{ }^{\circ} \mathrm{C}$ for $15 \mathrm{~s}$, annealing at $55^{\circ} \mathrm{C}$ for $30 \mathrm{~s}$ and elongation at $68{ }^{\circ} \mathrm{C}$ for $2 \mathrm{~min}$; with a final elongation at $68{ }^{\circ} \mathrm{C}$ for $5 \mathrm{~min}$. One-tenth of the volume of the PCR product was analyzed by agarose gel electrophoresis and then the remaining part was purified using a QIAquick PCR Purification Kit (Qiagen).

\section{Sequencing procedure}

The purified PCR product was subjected to direct sequencing using a BigDye Terminator Cycle Sequencing Kit (Applied Biosystems) using ABI PRISM 310 (Applied Biosystems). The DNA sequences were aligned by 'DNASIS' version 3.0 (Hitachi).

\section{Phylogenetic analysis}

ITS1 nucleotide sequences were aligned by ClustalX software. Maximum parsimony phylogenetic analysis with 1000 replicates was performed by PAUP* 4.0.

Cloning of PCR products

The ITS region was amplified from total DNA using a tagged primer set of ER Swer 1F (AAA AGA ATT CGA GGT CGC GAG AAG TCC ACT) and BH Swer 1R (AAA ACC ATG GCT CAG CGG GTA ATC CCG CCT). After purification using a QIAquick PCR purification kit, the PCR products (100-200 ng) were digested with EcoRI and BamHI (Takara Biotech) in a $10-\mu$ reaction mixture containing $10 \mathrm{mM}$ Tris-HCl, $\mathrm{pH} 7.5,10 \mathrm{mM} \mathrm{MgCl} 2,1 \mathrm{mM}$ dithiothreitol, and $50 \mathrm{mM} \mathrm{NaCl}$ at $37^{\circ} \mathrm{C}$ for $1 \mathrm{~h}$, and the reaction mixture was heated at $60{ }^{\circ} \mathrm{C}$ for $15 \mathrm{~min}$. Four microliters of the reaction mixtures were combined with $5 \mathrm{ng}$ of plasmid Bluescript SK (-) digested with the same enzymes and $5 \mu \mathrm{l}$ of DNA ligation solution Ver. 2.1 (Takara Biotech). The ligation reaction was performed at $16{ }^{\circ} \mathrm{C}$ overnight. Competent cells (Competent high DH5 $\alpha$, Toyobo) were transformed with the ligated product as recommended in the manufacturer's protocol. The transformed

Table 1 Materials used in this study

\begin{tabular}{lllll}
\hline Sample & Species & Locality & Date & Status (ID) \\
\hline $\mathbf{1}$ & Swertia japonica & Aokimura, Nagano, Japan & September 2007 & Cultivated plant (071009) \\
$\mathbf{2}$ & S. decora & Dali, Yunnan, China & September 2001 & Crude drug (KANP7581) \\
$\mathbf{3}$ & S. binchuanensis & Dali, Yunnan, Chian & October 2005 & Crude drug (KANP7582) \\
$\mathbf{4}$ & S. punicea & Dali, Yunnan, China & September 2001 & Crude drug (KANP7583) \\
$\mathbf{5}$ & S. delavayi & Dali, Yunnan, China & October 2005 & Crude drug (KANP7584) \\
$\mathbf{6}$ & S. pseudochinensis & Hebei, China & 2005 & Crude drug (KANP7585) \\
$\mathbf{7}$ & S. japonica & Nagano, Japan & 2005 & Crude drug (KANP7586) \\
\hline
\end{tabular}


Table 2 Nucleotide differences in ITS1, 5.8S ribosomal RNA gene, and ITS2

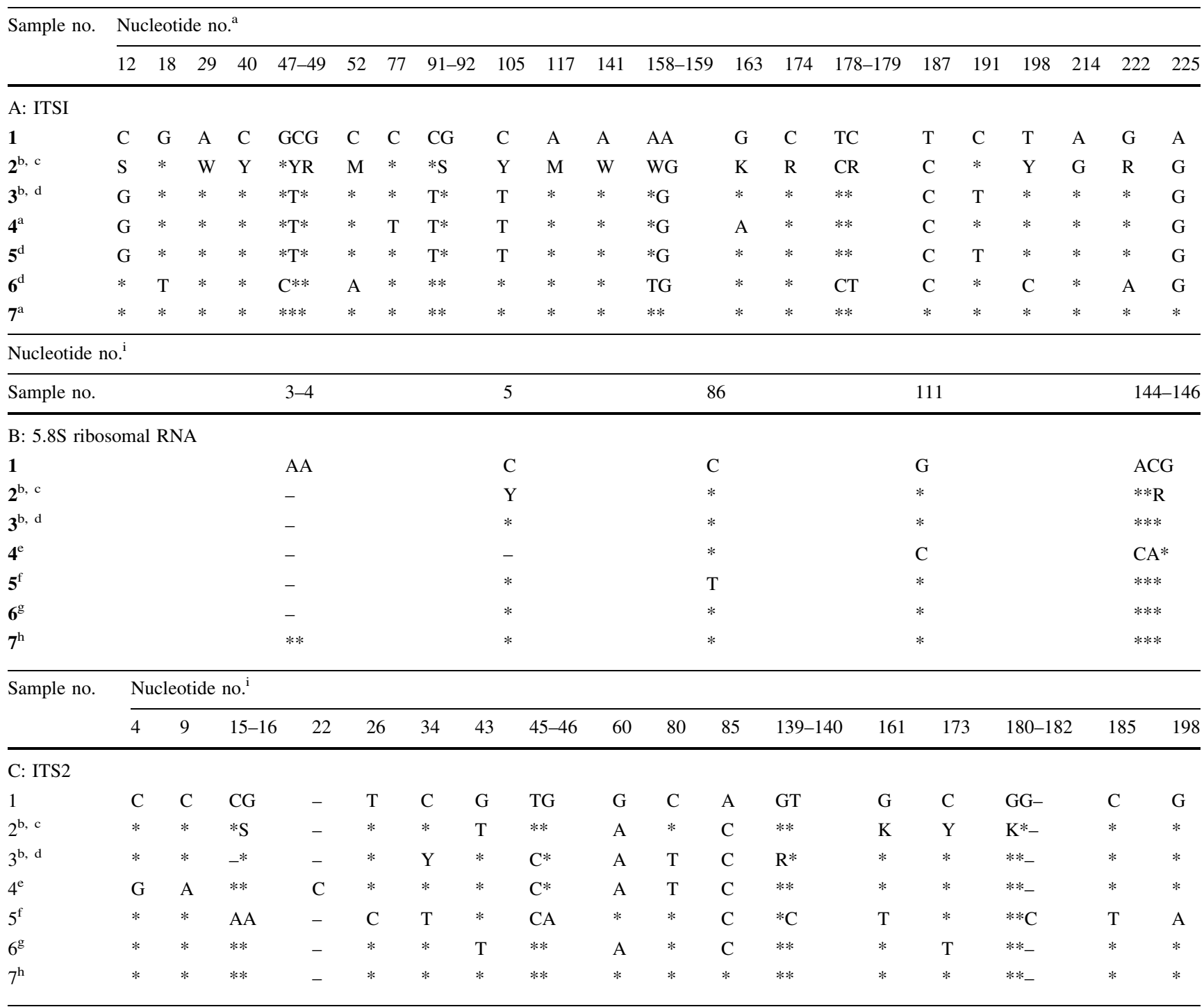

$S \mathrm{C}+\mathrm{G}, W \mathrm{~A}+\mathrm{T}, Y \mathrm{C}+\mathrm{T}, R \mathrm{~A}+\mathrm{G}, M \mathrm{~A}+\mathrm{C}, K \mathrm{G}+\mathrm{T}$

${ }^{a}$ Numbering is based on Hagen et al. (2001) [6]

${ }^{\mathrm{b}}$ Sequence data deduced from those of clones

c GenBank Accession No. GQ848483

d GenBank Accession No. GQ848484

e GenBank Accession No. GQ848485

${ }^{\mathrm{f}}$ GenBank Accession No. GQ848486

g GenBank Accession No. GQ848487

${ }^{\text {h }}$ GenBank Accession No.GQ848488

${ }^{i}$ Numbering is based on Liu et al. (2001) [7]

cells were spread on LB/Amp plates (2\% tryptone, $1 \%$ yeast extract, $2 \% \mathrm{NaCl}, 1.5 \%$ agar, and $100 \mu \mathrm{g} / \mathrm{ml}$ ampicillin) and incubated at $37{ }^{\circ} \mathrm{C}$ overnight. Bacterial colonies were picked up separately, and sub-cultured in liquid LB/Amp medium. After overnight incubation, the bacteria were collected by centrifugation and plasmids were obtained following the standard protocol [5].

\section{Results and discussion}

Direct sequencing of PCR products of the ITS region of samples $\mathbf{1}, \mathbf{4}, \mathbf{5}, \mathbf{6}$, and 7 was successful, while that of samples $\mathbf{2}$ and $\mathbf{3}$ gave ambiguous sequencing results caused by overlapping of divergent sequences of the respective samples. The PCR products of these 2 samples were 


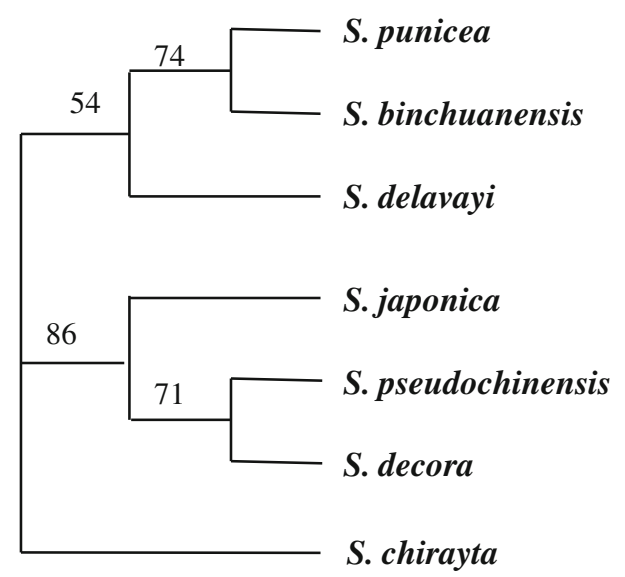

Fig. 1 Phylogenetic analysis of ITS1, 5.8S ribosomal RNA gene, and ITS2 of Yunnanese Swertia plants together with those of Japanese $S$. japonica and $S$. pseudochinensis from Hebei Province. The sequence data of $S$. chirayta (GenBank Accession No. AF255917) are used as an outgroup. The $50 \%$ majority-rule consensus tree was based on maximum parsimony analysis. Numbers above lines are bootstrap values (\%) with 1000 replicates

subjected to cloning analysis using an E. coli system, and the clones were sequenced. The sequence data of samples 2 and $\mathbf{3}$ were deduced from their 10 or 6 clones, respectively. The data obtained from direct sequencing as well as that deduced from clones are shown in Table 2, where the ITS1, 5.8S ribosomal RNA gene, and ITS2 sequences are aligned with those of S. japonica [6] or S. chirayta [7]. Maximum parsimony analysis using PAUP* 4.0 ver. 10 (Altivec, Sinauer Assoc. Inc.) was performed on the combined sequence of ITS1, 5.8S ribosomal RNA gene, and ITS2 together with that of $S$. chirayta as an outgroup (Figs. 1,2). The phylogenetic tree shows that two of the Yunnanese Swertia plants, S. binchuanensis and S. punicea, were genetically similar, and so were $S$. decora and S. pseudochinensis. The other Yunnanese Swertia plants, $S$. delavayi, and $S$. japonica, stood at intermediate positions between these two genetically similar pairs. The similarity in chemical constituent profiles of S. binchuanensis and $S$. punicea was correlated with their genetic background. On the other hand, although S. decora and S. pseudochinensis showed differences in chemical constituent profile and had relatively distant habitats, their genetic structure seemed similar. To survey the phylogenetic structure of Swertia plants growing in a wider range, further phylogenetic analysis was performed on the ITS1 data obtained from the present study and the database of Swertia plants distributed in Asia. Similar to the previous finding obtained from combined ITS1, 5.8S ribosomal RNA gene, and ITS2 sequences, $S$. decora and S. pseudochinensis were positioned in the same clade, but not in the clade of the other plants from Yunnan, S. binchuanensis, S. delavayi, and $S$. punicea. In addition, the position of $S$. decora and

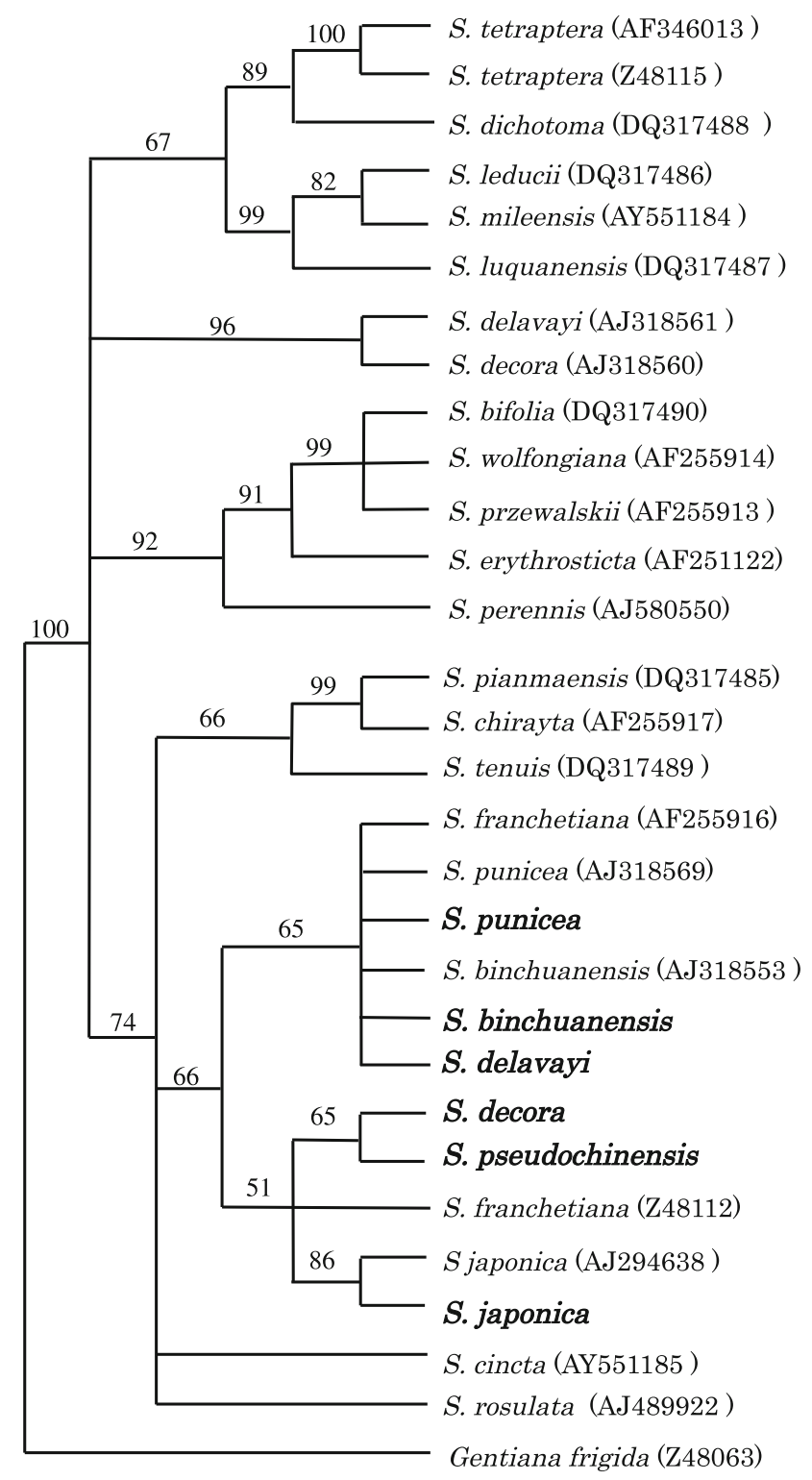

Fig. 2 Phylogenetic analysis of ITS1 sequences of Swertia plants. The sequence data of Gentiana frigida (GenBank Accession No. Z48063) are used as an outgroup. The $50 \%$ majority-rule consensus tree was based on maximum parsimony analysis. Numbers above lines are bootstrap values (\%) with 1000 replicates. Species shown in bold are the results of this study. Data from GenBank are shown with accession numbers in parentheses

S. delavayi in this study was inconsistent with that reported by Chassot et al. [8]. Re-examination of both of the materials might be necessary. However, Swertia plants from Yunnan, Hebei, and Japan analyzed in this study were phylogenetically close in comparison with Swertia plants obtained from Qinghai and Tibet (S. bifolia, S. przewalskii, and S. erythrosticta) [9] and from South Asia including India and Nepal (S. chirayta) [8]. The result of our study suggests that although genetic background would have an influence, environmental factors, e.g., soil and weather 
conditions, might be critical for their production of secondary metabolites.

\section{References}

1. Basnet P, Kadota S, Namba T, Shimizu M (1994) Bellidifolin: a potent hypoglycemic agent in streptozotocin (STZ)-induced diabetic rats from Swertia japonica. Planta Med 60:507-511

2. Hase K, Li J, Basnet P, Xiong Q, Takamura S, Namba T, Kadota S (1997) Hepatoprotective principles of Swertia japonica Makino on D-galactosamine/lipopolysaccharide-induced liver injury in mice. Chem Pharm Bull 45:1823-1827

3. Yamahara J, Kobayashi M, Matsuda H, Aoki S (1991) Anticholinergic action of Swertia japonica and an active constituent. J Ethnopharmacol 33:31-35

4. Wang Z, Ma C, Tang S, Xiao H, Kakiuchi N, Kida H, Hattori M (2008) Qualitative and quantitative analysis of Swertia herbs by high performance liquid chromatography-diode array detectormass spectrometry (HPLC-DAD-MS). Chem Pharm Bull 56: 485-490

5. Sambrook J, Fritsch EF, Maniatis T (eds) (1989) Chapter 1, molecular cloning, 2nd edn. Cold Spring Harbor Press, Cold Spring Harbor, New York

6. Hagen KBV, Kadereit JW (2001) The phylogeny of Gentianella (Gentianaceae) and its rapid diversification in the southern hemisphere as revealed by nuclear and chloroplast DNA sequence variation. Org Divers Evol 1:61-79

7. Liu JQ, Chen ZD, Liao ZX, Lu AM (2001) A comparison of the its sequences of the Tibetan medicine 'zang yin chen'-Swertia mussotti and its adulterant species. Yao Xue Xue Bao 36:67-70

8. Chassot P, Nemomissa S, Yuan YM, Kupfer P (2001) High paraphyly of Swertia L. (Gentianaceae) in the Gentianella-lineage as revealed by nuclear and chloroplast DNA sequence variation. Plant Syst Evol 229:1-21

9. Xue CY, Li DZ, Lu JM, Yang JB, Liu JQ (2006) Molecular authentication of the traditional Tibetan medicinal plant Swertia mussotii. Planta Med 72:1223-1226 